\title{
Regeneración natural de sitios de manglar degradado en respuesta a la restauración hidrológica
}

\author{
Natural regeneration of degraded mangrove sites in response to \\ hydrological restoration
}

\author{
Stephanie Echeverría-Ávila1,3, Rosela Pérez-Ceballos²*, Arturo Zaldívar-Jiménez1, Julio Canales-Delgadillo², \\ Roberto Brito-Pérez ${ }^{3}$, Martín Merino-Ibarra ${ }^{4}$ y Alejandra Vovides ${ }^{5}$
}

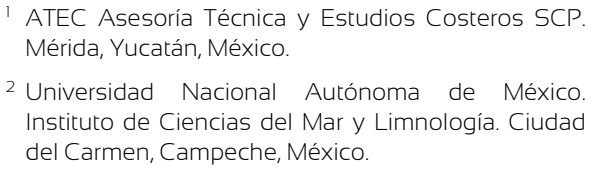

\author{
Universidad Autónoma del Carmen. Centro de \\ Ciencias Ambientales. Ciudad del Carmen, \\ Campeche, México. \\ ${ }^{4}$ Universidad Nacional Autónoma de México. \\ Instituto de Ciencias del Mar y Limnología. Unidad
}

\author{
Académica de Ecología y Biodiversidad Acuática. \\ Ciudad de México, México. \\ ${ }^{5}$ University of Glasgow. School of Geographical and \\ Earth Science. Glasgow, Reino Unido. \\ * Autora de correspondencia. rosela.perezc@agmail.com
}

\section{RESUMEN}

Los manglares de Laguna de Términos, Campeche han sido afectados por la pérdida de la conexión hidrológica durante el paso de tormentas y huracanes. El objetivo de este trabajo fue evaluar la regeneración natural del manglar en un área degradada, después de haber implementado acciones de restauración hidrológica. Se establecieron tres sitios de muestreo con diferentes tiempos de restauración (1 año - 3 años) y un sitio de manglar natural conservado como referencia. El muestreo se realizó en los años 2014 a 2015. Se evaluó densidad, altura, sobrevivencia y tasa de crecimiento de las plántulas, así como las características ambientales. La relación entre la vegetación y las características ambientales se realizaron mediante regresiones lineales simples y un análisis de escalamiento multidimensional. Para determinar la dispersión de propágulos respecto a los manglares naturales, se generó un modelo lineal mixto de la densidad de propágulos $v s$. la distancia y el tiempo de restauración. Los resultados demostraron que la distancia respecto a los manglares naturales afectó la densidad de los propágulos. La concentración de fosfato se relacionó con la sobrevivencia de las plántulas; mientras que la concentración de amonio y la concentración de sulfuro se relacionaron negativamente con la densidad de las plántulas. Las acciones de restauración hidrológica influyeron en el establecimiento y desarrollo de la vegetación de manglar; por lo tanto, las zonas de manglar natural y la restauración hidrológica facilitaron la dispersión de los propágulos hacia las zonas degradadas, resaltando la importancia de contemplar estas áreas naturales dentro de los proyectos de restauración.

PALABRAS CLAVE: Aviccenia germinas, crecimiento, estructura forestal, evaluación ambiental, plántulas de manglar.

\section{ABSTRACT}

The mangroves of Laguna de Términos, Campeche have been affected by the loss of hydrological connection due to tropical storms and hurricanes. The objective of this study was to evaluate the natural regeneration in a degraded area, after having implemented actions of restoration through hydrological restoration. Three sampling sites with different times of restoration (1-3 years), and a preserved natural mangrove site as reference, were established. The sampling was carried out in the years 2014 to 2015. Forest structure (density, height), survival, growth rate of seedlings and environmental characteristics were measured. The relationship between the vegetation and environmental characteristics was determined using simple linear regressions and multidimensional scaling analysis. To determine the dispersal of seedlings, a mixed linear model of the seedling density $v$ distance $v s$ time of restoration was generated. The results showed that distance to natural mangroves affects the seedling density. The phosphates concentration is related to survival, while the ammonia and sulfide concentration is negatively related to seedling density. The actions of hydrological restoration influenced the settlement and growth seedling of mangrove vegetation, therefore natural areas of mangroves and the water reconnection facilitated propagule dispersal towards the degraded areas, highlighting the importance of the inclusion of natural areas within the restoration projects.

KEYWORDS: Aviccenia germinans, growth, forest structure, environmental evaluation, mangrove seedlings. 


\section{INTRODUCCIÓN}

La franja costera de las regiones tropicales está dominada frecuentemente por los manglares, uno de los ecosistemas biológicamente más importantes por su alta productividad primaria y secundaria (Barbier, 2016; Kuenzer, Bluemel, Gebhardt, Quoc y Dech, 2011). Su estructura forestal brinda bienes y servicios ecosistémicos como la captura de carbono, estabilización de la línea de costa y protección de las comunidades costeras contra la marea y oleaje generados por tormentas (Gedan, Kirwan, Wolanski, Barbier y Silliman, 2011); además, es hábitat de aves que anidan, se alimentan y se resguardan en ellos (Buelow y Sheaves, 2015).

La estructura forestal de los manglares se caracteriza por atributos como la riqueza de especies, la altura, el área basal; así como el desarrollo del sotobosque (Lugo y Snedaker, 1974; Tomlinson, 1986). En la Laguna de Términos se encuentran las especies Rhizophora mangle, Avicennia germinans y Laguncularia racemosa; encontrándose manglares tipo cuenca, tipo franja y tipo ribereño. Los manglares de la Isla del Carmen, ubicada dentro de la Laguna de Términos, se han caracterizado por presentar altura del dosel entre $5 \mathrm{~m}$ y $12.5 \mathrm{~m}$, el área basal entre 8.6 $\mathrm{m}^{2} \mathrm{ha}^{-1}$ y $9.9 \mathrm{~m}^{2} \mathrm{ha}^{-1}$ y la densidad de árboles entre 1670 y 3095 árboles por hectárea (Coronado-Molina et al., 2012). Los procesos ecológicos determinantes de la estructura y función de los manglares están condicionados principalmente por el hidroperíodo (la frecuencia, el nivel y la duración de las inundaciones), por la disponibilidad de nutrientes en el suelo (Twilley y Rivera-Monroy, 2005) y por las variaciones de salinidad, temperatura, potencial de óxido reducción y concentración de sulfuro, que actúan como reguladores del crecimiento de este tipo de vegetación (Lewis et al., 2015).

La alteración del hidroperíodo por la pérdida de la conectividad hidrológica entre un cuerpo de agua y una zona de manglar ocasiona el incremento en la salinidad del suelo y la temperatura del agua, afectando la estructura forestal del ecosistema al disminuir el vigor y la densidad de las especies del manglar (Lewis et al., 2015), lo que conlleva a una marcada reducción en las concentraciones de oxígeno, un aumento de la concentración de sulfuros y alteraciones en los ciclos de los nutrientes (McKee, Mendelssohn y Hester, 1988; Vovides, Bashan, LópezPortillo y Guevara, 2011). Lo anterior resulta en la rápida degradación de la vegetación y pérdida de las funciones del ecosistema.

Dentro de las estrategias de manejo de los manglares, la restauración ecológica es un componente clave para la sostenibilidad a largo plazo, especialmente cuando están altamente degradados o amenazados (Aronson y Alexander, 2013). Específicamente la restauración hidrológica, mediante el desazolve de los canales de marea, permite el flujo de agua hacia zonas degradadas y el acarreo de propágulos desde fuentes de germoplasma (Hastuti, Anggoro y Pribadi, 2012; Vogt et al., 2014). La restauración hidrológica modifica el hidroperíodo y ha demostrado ser el método más eficiente para mejorar la calidad del suelo en zonas de manglar en México (Pérez-Ceballos, EcheverriaAvila, Zaldívar-Jiménez, Zaldívar-Jiménez y HerreraSilveira, 2017; Zaldívar-Jiménez, Guevara, Pérez-Ceballos, Díaz y Rosado, 2017), brindando condiciones biogeoquímicas favorables para facilitar los procesos de reclutamiento de propágulos provenientes de la vegetación adyacente con escaso o nulo disturbio (Duke, 2001; Sousa, Quek y Mitchell, 2003). En otros países la restauración hidrológica también ha sido un método exitoso, por ejemplo en Florida, EE.UU. (Turner y Lewis III, 1997), Colombia (Jaramillo et al., 2018) e Indonesia (Brown, Fadillah, Nurdin, Soulsby y Ahmad, 2014).

La evolución y el éxito de las actividades de restauración hidrológica aplicadas a zonas degradadas, pueden evaluarse a través del monitoreo de características como la estructura de la vegetación y del suelo (Salmo, Lovelock y Duke, 2013). Estos indicadores deben ser evaluados en diferentes niveles temporales de la restauración (Zhao et al., 2016).

\section{OBJETIVOS}

Los objetivos de la presente investigación fueron: 
1. Evaluar la influencia de la restauración hidrológica sobre la regeneración natural de manglares en restauración.

2. Analizar la dispersión, reclutamiento y crecimiento de la regeneración de manglar en las zonas de restauración.

3. Identificar la relación de factores ambientales sobre la regeneración natural del manglar en diferentes etapas de la restauración hidrológica.

\section{MATERIALES Y MÉTODOS}

\section{Área de estudio}

El área de estudio se localiza en Isla del Carmen, Campeche; al sur del Golfo de México, dentro del área natural protegida Laguna de Términos $18^{\circ} 40^{\prime} 18.37^{\prime \prime} \mathrm{N}, 91^{\circ} 40^{\prime}$ 24.93" O (Fig. 1). La Laguna de Términos es considerada como el sistema lagunar estuarino de mayor volumen y extensión en México con 20114.10 ha (Comisión Nacional para el Conocimiento y Uso de la Biodiversidad [Conabio], 2007), además de ser un área de protección de flora y fauna (Secretaría de Desarrollo Social [Sedesol], 1994) y sitio Ramsar como humedal de importancia internacional (Venegas, 2003).

Dentro del sistema lagunar de Términos se localiza la Isla del Carmen que es un isla de barrera constituida principalmente por carbonatos de calcio (Bach et al., 2005), con un sistema deltaico, bordeada principalmente por vegetación de manglar y pequeñas lagunas asociadas. La distribución y condición de los manglares de la Isla del Carmen están determinados por las mareas (CoronadoMolina et al., 2012), donde la amplitud vertical del intervalo de marea registrado fue de $0.34 \mathrm{~m}$ (Contreras, Douillet y Zavala-Hidalgo, 2014). El clima es tropical húmedo y se presentan tres estaciones climáticas: la estación seca de febrero a abril, la estación de lluvias de junio a septiembre y la temporada de frentes fríos de octubre a marzo (Guerra y Kahl, 2017).

El área de estudio fue una zona de manglar con pérdida de conectividad hidrológica debido al bloqueo por árboles derribados sobre los canales de marea durante el paso de los huracanes Opal y Roxana en el año 1995. Principalmente en el año 2010, se realizó la restauración hidrológica, mediante el desazolve de $7826 \mathrm{~m}$ lineales de canales de marea y la modificación de la topografía, con el propósito de restaurar 160 ha de manglares (ZaldívarJiménez et al., 2017). Las acciones de restauración hidrológica y mantenimiento han sido realizadas por la comunidad de Isla Aguada (Carmen, Campeche) y se han continuado hasta el año 2017.

\section{Diseño del muestreo}

Se establecieron cuatro sitios de muestreo: tres localizados dentro de la zona de restauración hidrológica y uno como sitio de referencia (control) en un manglar natural de una zona adyacente al área degradada. Los tres sitios ubicados en la zona de restauración se categorizaron en función de las acciones de la restauración que fueron aplicadas: sitio 1 (dos años después), sitio 2 (tres años), sitio 3 (cuatro años) y el sitio 4 fue establecido como referencia. La evaluación de la estructura forestal para los sitios en restauración se realizó en las zonas adyacentes. Para la evaluación de la regeneración natural dentro de las zonas en restauración, tomando como criterio la variación de los niveles de inundación, se llevaron a cabo tres muestreos durante un año del 2014 al 2015. El primer muestreo se realizó en el mes de mayo (mínimas inundaciones), el segundo en octubre (máximas inundaciones) y el tercero en febrero (inundación intermedia). Además, se evaluaron las características ambientales de cada sitio donde se midieron los fisicoquímicos, los nutrientes inorgánicos del agua del suelo y la densidad aparente del suelo.

\section{Características biológicas}

Estructura forestal de la zona adyacente. En cada sitio se establecieron dos unidades de muestreo de $10 \mathrm{~m} \times 10 \mathrm{~m}$, en las que se midió el diámetro a la altura del pecho (dap; $\mathrm{cm})$ y la altura $(\mathrm{m})$ de todos los árboles con diámetros mayores a $2.5 \mathrm{~cm}$. Se calculó el área basal $\left(\mathrm{m}^{2} \mathrm{ha}^{-1}\right)$, la densidad (árbol ha-1) y el índice de valor de importancia (VI) de cada especie forestal presente (Curtis y McIntosh, 1951). 


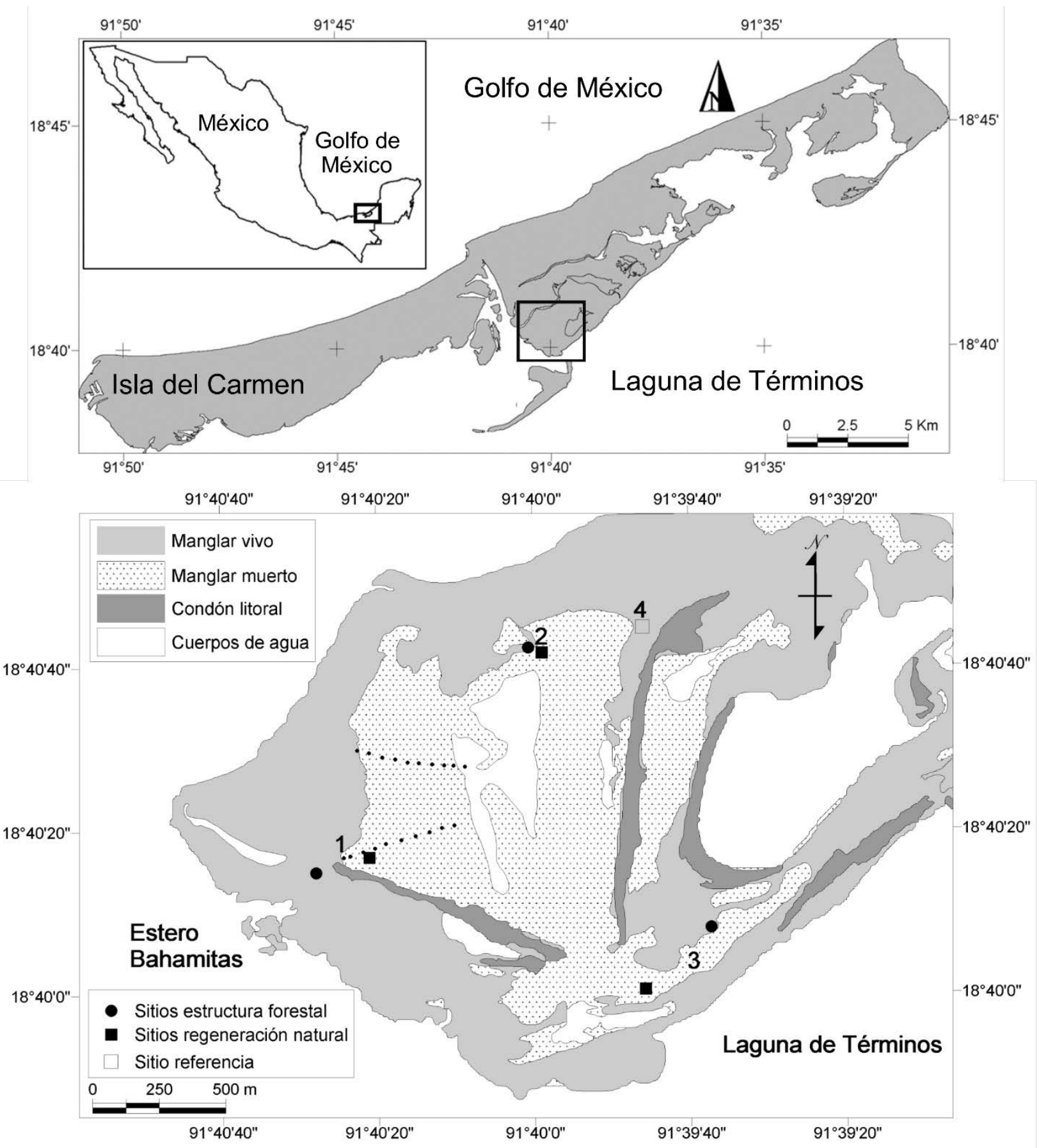

FIGURA 1. Localización de los sitios de muestreo dentro del área de estudio.

Regeneración natural en las zonas de restauración. Para estimar el reclutamiento se establecieron dos subunidades permanentes de muestreo de $1 \mathrm{~m}^{2}$, dentro de las cuales se registraron todas las plántulas presentes, a estas se les colocó una placa de identificación individual para dar seguimiento a los cambios en altura $(\mathrm{cm})$ y calcular la tasa relativa de crecimiento en altura (TRCA, $\left.\mathrm{cm} \mathrm{día}^{-1}\right)$ con la fórmula: TRCA $=\left(\mathrm{H}_{2}-\mathrm{H}_{1}\right) / \mathrm{n}$, donde: $\mathrm{n}=$ número de días entre mediciones, $\mathrm{H}_{1}=$ altura de la planta en el tiempo $1, \mathrm{H}_{2}=$ altura de la planta en el tiempo 2 (Rodríguez, Nivia y Garzón, 2004). También la sobrevivencia (\%) se calculó con las plántulas marcadas, por diferencia del número de 
plántulas vivas en el $T_{2}$ menos las registradas en el $T_{1}$. La densidad de las plántulas se calculó sobre una parcela de 25 $\mathrm{m}^{2}$ para disminuir el error de sobre estimación y posteriormente se convirtió a densidad por cada 0.01 ha, para fines comparativos.

Dispersión y reclutamiento de plántulas en la zona de restauración. Para determinar si las zonas adyacentes (sin disturbio) a la zona degradada eran importantes para la regeneración natural, se realizaron tres muestreos en los años de 2014 al 2016 sobre dos transectos de $450 \mathrm{~m}$ cada uno, desde las zonas de manglares naturales hacia los sitios en restauración. En cada transecto se establecieron estaciones separadas a una distancia de $50 \mathrm{~m}$ entre ellas, se estimó la densidad de plántulas de cada especie como una medida de la dispersión de los propágulos producida por la fuerza hidrológica.

\section{Características ambientales}

Para determinar la densidad aparente del suelo, se extrajeron dos muestras de suelo en cada sitio con un nucleador de acero inoxidable de $10 \mathrm{~cm}$ de diámetro y 30 $\mathrm{cm}$ de alto. La determinación se realizó a tres diferentes profundidades $(0 \mathrm{~cm}-10 \mathrm{~cm}, 10 \mathrm{~cm}-20 \mathrm{~cm}$ y $20 \mathrm{~cm}-30$ $\mathrm{cm})$, analizándose mediante la técnica descrita por Chen y Twilley (1999).

Para las muestras del agua intersticial, se extrajo agua del suelo a una profundidad de $30 \mathrm{~cm}$ usando jeringas y tubos acrílicos. Se midió in situ la salinidad y la temperatura del agua del suelo, se colocaron tres muestras de agua colectadas en cada sitio, utilizando una sonda multiparamétrica (YSI modelo-30). El Eh se midió utilizando un electrodo HI9126 (HANNA) que fue corregido añadiendo + 222 (Bohn, 1971). La determinación de las concentraciones de los iones se realizó in situ, por lo que no se requirió fijar la muestra. La determinación de los nitritos se hizo con el método del clorante azoico, los nitratos se determinaron por el método de reducción de cadmio, para el amonio se empleó el método de nesslerización directa, los fosfatos se determinaron mediante el método de cloruro de estaño y los sulfuros utilizando el método azul de metileno (American Public Health Association [APHA], 2005). Estas determinaciones se realizaron utilizando un kit de prueba para cada ion y un V-2000 multi-analyte photometer (CHEMetrics, Inc., Calverton, VA, USA).

La determinación del hidroperíodo se realizó en cada sitio, instalando un medidor de presión (HOBO, Onset Corporation) que midió cada hora de manera continua durante un año, de marzo de 2014 a febrero de 2015.

\section{Análisis estadísticos}

La variación de los datos medidos entre sitios y tiempo de muestreos no cumplió con el supuesto de normalidad, por lo que se analizaron a través una prueba de Kruskal Wallis. Se aplicó una prueba a posteriori de Tukey y Kramer (Nemenyi). La asociación entre las variables biológicas de las plántulas (densidad, altura, tasa de crecimiento y sobrevivencia) con las ambientales (salinidad, temperatura, Eh, $\mathrm{pH}, \mathrm{NO}_{3}^{-}+\mathrm{NO}_{2}^{-}, \mathrm{PO}_{4}^{-3}, \mathrm{NH}_{4}^{+}$y $\mathrm{S}^{-2}$ ) se analizó mediante regresiones lineales simples. Estos análisis se llevaron a cabo utilizando R 2.11.1 (R Development Core Team, 2008).

Las semejanzas entre los sitios se analizaron mediante el escalado multidimensional no métrico (NMDS), con el fin de identificar grupos que compartieran características, además de los cambios y tendencias que presentaron con respecto a las etapas de restauración. El NMDS mide la distancia entre objetos; la interpretación del gráfico depende de qué tan bajo es el valor del estrés. Los objetos unidos por los tramos más cortos son los más cercanos y los separados por lapsos más largos son más lejanos (Clarke y Ainsworth, 1993; Quinn y Keough, 2002). Para este análisis se utilizó una matriz de datos biológicos transformados $(\log V+1)$ y normalizados. Este análisis se llevó a cabo mediante el programa estadístico Primer v6.

La dispersión y reclutamiento de las plántulas en la zona de restauración se analizó utilizando un modelo lineal mixto tomando como variable de respuesta la densidad de plántulas. La densidad de las especies fue usada como efectos fijos y la distancia y el año como efectos aleatorios. 
Este análisis se llevó a cabo utilizando R 2.11.1 (R Development Core Team, 2008).

\section{Resultados}

\section{Características biológicas}

El desarrollo de la vegetación que bordea la Laguna de Términos tiene como especie dominante Avicennia germinans. El sitio 1, con dos años en restauración, registró las especies: Avicennia germinans, Rhizophora mangle y Laguncularia racemosa con densidad de 3800 individuos por hectárea (ind ha ${ }^{-1}$ ) en total. El sitio 2 registró menor densidad con 1450 ind ha- ${ }^{-1} \mathrm{y}$ menor dap con valor de $1.86 \mathrm{~cm}$. La mayor altura se registró en el sitio 4 con $6.86 \mathrm{~m}$ (Tabla 1 ).

Se encontraron diferencias significativas en la densidad de plántulas entre sitios (Kruskal-Wallis $\chi^{2}=19.647$, g.l. $=$ $3, \mathrm{p}<0.001)$. El sitio 4 presentó una mayor densidad promedio respecto a los sitios 2 y 3 (prueba de Nemenyi, $\mathrm{p}$ $=0.001)$. Respecto a la altura, los sitios difirieron significativamente (Kruskal-Wallis $\chi^{2}=9.280$ g.l. $=3, \mathrm{p}=$ 0.02 ), específicamente entre los sitios 3 y 4 (prueba de Nemenyi, $p=0.017)$. La tasa de crecimiento presentó diferencia significativa (Kruskal-Wallis $\chi^{2}=3.182$ g.l. $=3, \mathrm{p}$ $=0.036)$. El porcentaje de sobrevivencia no tuvo diferencias entre sitios (Kruskal-Wallis $\chi^{2}=1.745$, g.l. $=3, \mathrm{p}=0.062$ ). En el sitio 1 se registraron 148 plántulas en 0.01 ha, de las cuales $A$. germinans registró $109, \mathrm{R}$ mangle 32 y L racemosa presentó menor densidad con 7 plántulas. En este sitio también se presentó más crecimiento, registrándose alturas de $64.22 \mathrm{~cm}$ y presentando mayores valores de TRCA con
$0.15 \mathrm{~cm}$ día $^{-1}$. El sitio 2 presentó menor densidad con 26 plántulas en 0.01 ha de A. germinans; mientras que el sitio 3 presentó una densidad de 79 plántulas, de las cuales 78 correspondieron a $A$. germinans y solamente un individuo correspondió a $R$. mangle; en este sitio se observó el mayor porcentaje de sobrevivencia con $88 \%$. El sitio 4 presentó la mayor densidad con 783 plántulas en 0.01 ha, de las cuales 25 eran de R. mangle y el resto de A. germinans; sin embargo, fue el sitio con menor altura con $32.59 \mathrm{~cm}$ (Tabla 2).

En los transectos se observó que existió mayor densidad de plántulas de $A$. germinans en los primeros $100 \mathrm{~m}$ de distancia con respecto al manglar natural. La especie R. mangle presentó una densidad menor a 50 plántulas en 0.01 ha en los primeros $150 \mathrm{~m}$ y después de esta distancia disminuyó su densidad $(t=-2.40, p>0.038, d f=9.38)$. La especie $L$. racemosa registró la menor densidad y se encontró únicamente en los primeros $50 \mathrm{~m}$ del transecto $(\mathrm{t}=-2.62, \mathrm{p}>0.026, \mathrm{df}$ = 9.34). El modelo lineal mixto sugirió que la distancia a la cual se establecieron las plántulas tuvo un efecto negativo sobre la densidad $(t=5.279, \mathrm{p}<0.001)$, estimando que $L$. racemosa y $\mathrm{R}$. mangle disminuyeron su densidad respecto a la distancia en un intervalo entre 26 y 29 plántulas en 0.01 ha por cada $50 \mathrm{~m}$ de distancia respecto al manglar natural (Fig. 2). El factor año tuvo un efecto positivo respecto al incremento en la densidad plántulas de manglar, este efecto fue pequeño y estadísticamente marginal $(\mathrm{t}=1.81, \mathrm{p}=0.07)$. Por otro lado, se observó que a la distancia de $350 \mathrm{~m}$ se incrementó la densidad de $A$. germinans de 95 a 197 plántulas en 0.01 ha, debido a su cercanía con parches de manglar remanentes en el área de restauración.

TABLA 1. Estructura forestal de los árboles adultos en las zonas adyacentes a los sitios de restauración (sitios de 1 al 3 ) y el manglar de referencia (sitio 4).

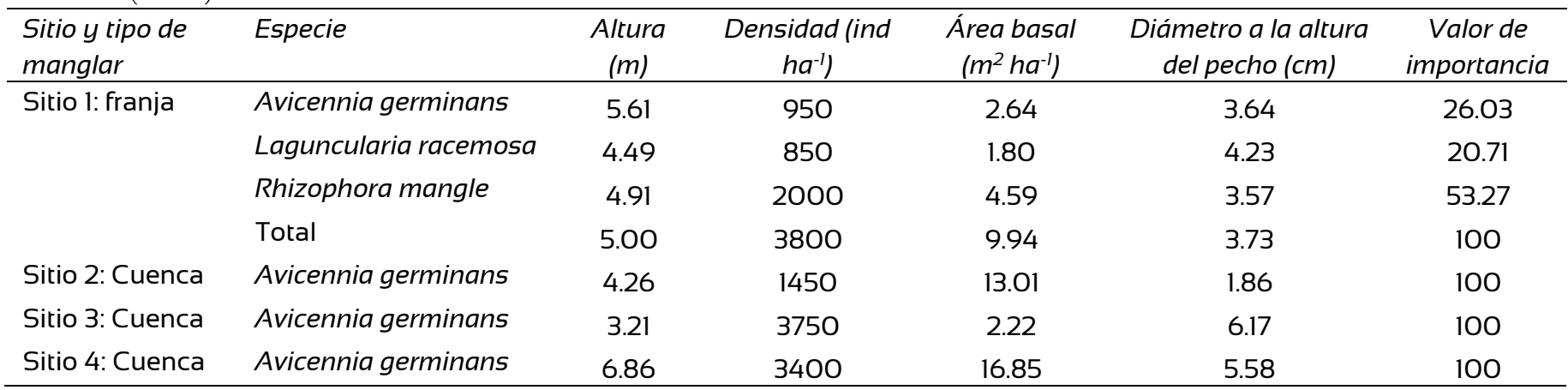


TABLA 2. Características biológicas de la regeneración natural de los sitios en restauración (sitios 1, 2 y 3) y el sitio de referencia (sitio 4) en el estero de Laguna de Términos.

\begin{tabular}{lccccc}
\hline Variables & Sitio 1 & Sitio 2 & Sitio 3 & Sitio 4 & Valor de P \\
\hline Densidad (individuos en O.01 ha) & $148 \pm 22.80$ & $26 \pm 4.93$ & $79 \pm 19.12$ & $783 \pm 197.24$ & $<0.001$ \\
Altura (cm) & $64.22 \pm 23.99$ & $39.04 \pm 3.97$ & $55.36 \pm 3.36$ & $32.59 \pm 1.91$ & 0.002 \\
TRCA $(\mathrm{cm} \mathrm{dia-1})$ & $0.15 \pm 0.01$ & $0.03 \pm 0.01$ & $0.03 \pm 0.01$ & $0.05 \pm 0.01$ & 0.036 \\
Sobrevivencia (\%) & $80.91 \pm 8.48$ & $80.56 \pm 9.04$ & $87.84 \pm 4.59$ & $69.28 \pm 10.88$ & 0.062 \\
\hline
\end{tabular}

Los valores indican la media \pm error estándar. La última columna representa diferencias significativas a nivel ( $\mathrm{P} \leq 0.05)$. Los valores de Kruskal-Wallis corresponde el análisis entre sitios. TRCA: tasa relativa de crecimiento en altura.

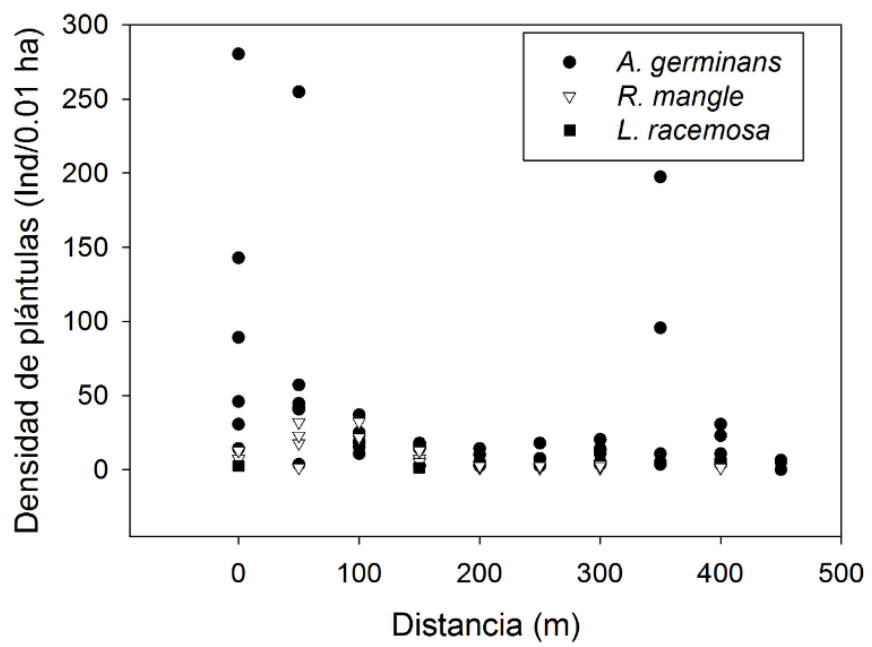

FIGURA 2. Efecto de la vegetación sin disturbio (los símbolos son la presencia de la vegetación) sobre la densidad de plántulas en las áreas en restauración.

\section{Características ambientales}

Se observó un patrón inverso de la densidad aparente del suelo con respecto al tiempo de restauración (Tabla 3). La salinidad no mostró diferencias significativas entre sitios, pero sí en los tiempos del muestreo (Kruskal-Wallis $\chi^{2}=$ 7.274, g.l. $=2, \mathrm{p}<0.026$ ), durante la temporada de mínimas inundaciones $(0.07 \mathrm{~m})$ se registraron 62.57 ups en el sitio 4 (prueba de Nemenyi, $\mathrm{p}=0.045)$. Los valores medios de salinidad para el agua intersticial en los sitios 1 a 3 (47.53 ups a 51.51 ups, respectivamente) fueron inferiores a los registrados en el sitio 4 (55.21 ups). La temperatura no presentó diferencias entre sitios y tiempo de muestreo. El sitio 2 presentó la temperatura más elevada con $30.77^{\circ} \mathrm{C}$. Los valores de Eh no presentaron diferencias entre sitios, ni entre tiempos. Los sitios 1 al 3, presentaron los valores de Eh negativos (máximo $=-61.20 \mathrm{mV}$ ), mientras que el sitio 4 registró solamente valores positivos (Tabla 3). El pH registró diferencias entre sitios y entre el tiempo del muestreo (Kruskal-Wallis $\chi^{2}=15.172$, g.l. $=2, \mathrm{p}<0.001$ ); la prueba de Tukey y Kramer (prueba de Nemenyi, p = $0.05)$ identificó el mayor $\mathrm{pH}$ en la temporada de inundaciones intermedias, con 6.44 para el sitio 3. Se observaron diferencias entre sitios y en tiempo $(\mathrm{p}<0.05)$ para las variables de $\mathrm{PO}_{4}^{-3}$ (fostatos) y $\mathrm{S}^{-2}$ (sulfuro) (prueba de Nemenyi, $\mathrm{p}=0.045)$, pero para las concentraciones de $\mathrm{NO}_{2}^{-}$(nitritos) $+\mathrm{NO}_{3}^{-}$(nitratos) y $\mathrm{NH}_{4}{ }^{+}$(amonio) no se presentaron diferencias entre sitios ni entre tiempos.

Con respecto a las variables del hidroperíodo, los sitios presentaron diferencias entre sitios respecto a la frecuencia de inundación (Kruskal Wallis $\chi^{2}=28.81$, g.l $=$ 3 , p $<0.001)$, también se observaron diferencias en el tiempo de inundación (Kruskal Wallis $\chi^{2}=22.63$, g.l $=3$, $\mathrm{p}<0.001$ ) y en el nivel de inundación (Kruskal Wallis $\chi^{2}=$ 20.53, g.l $=3, \mathrm{p}=0.001)$. Los sitios 3 y 4 , así como los sitios 1 y 2 , presentaron patrones del hidroperíodo similares entre ellos. Las mayores frecuencias de inundación fueron en los sitios 3 y 4 ( 23 y 24 inundaciones al mes respectivamente) durante el mes de septiembre. Los sitios 1 y 2 permanecieron inundados más de $600 \mathrm{~h}$ al mes, el sitio 1 presentó este comportamiento todos los meses del año y el sitio 2 lo presentó de junio 2014 a enero 2015. Los mayores niveles de inundación se registraron de septiembre 2014 a enero 2015 para todos los sitios, los sitios 1 y 2 fueron los que presentaron los mayores niveles con $0.16 \mathrm{~m}$ y $0.25 \mathrm{~m}$, respectivamente. Los sitios 3 y 4 presentaron niveles de inundación entre $0.084 \mathrm{~m}$ y $0.086 \mathrm{~m}$ en promedio, respectivamente (Tabla 4). 
TABLA 3. Características ambientales del suelo y el agua intersticial en Bahamitas, Laguna de Términos durante el período del estudio.

\begin{tabular}{|c|c|c|c|c|c|c|}
\hline Variables & Sitio 1 & Sitio 2 & Sitio 3 & Sitio 4 & $\begin{array}{c}\text { Kruskal- } \\
\text { Wallis test }\end{array}$ & $\begin{array}{c}\text { Valor de } \\
P\end{array}$ \\
\hline Densidad aparente $\left(\mathrm{g} \mathrm{cm}^{-3}\right)$ & $0.62 \pm 0.10$ & $0.30 \pm 0.05$ & $0.27 \pm 0.04$ & $0.23 \pm 0.02$ & 11.02 & 0.012 \\
\hline Salinidad (ups) & $47.53 \pm 7.04$ & $51.51 \pm 8.41$ & $48.08 \pm 2.11$ & $55.21 \pm 4.12$ & 3.11 & 0.374 \\
\hline Temperatura $\left({ }^{\circ} \mathrm{C}\right)$ & $28.84 \pm 1.10$ & $30.77 \pm 1.13$ & $27.97 \pm 0.55$ & $29.05 \pm 1.03$ & 6.62 & 0.085 \\
\hline Eh $(m V)$ & $-36.26 \pm 25.64$ & $-15.14 \pm 42.72$ & $-83.64 \pm 22.90$ & $18.11 \pm 35.9$ & 8.71 & 0.033 \\
\hline $\mathrm{pH}$ & $6.27 \pm 0.53$ & $6.23 \pm 0.45$ & $6.44 \pm 0.44$ & $6.12 \pm 0.13$ & 2.74 & 0.433 \\
\hline $\mathrm{S}^{-2}\left(\mathrm{mg} \mathrm{l}^{-1}\right)$ & $7.68 \pm 3.31$ & $8.54 \pm 5.55$ & $24.78 \pm 14.63$ & $16.31 \pm 5.21$ & 10.79 & 0.012 \\
\hline $\mathrm{NO}_{2}^{-}+\mathrm{NO}_{3}^{-}\left(\mathrm{mg} \mathrm{l}^{-1}\right)$ & $1.30 \pm 0.57$ & $1.37 \pm 0.32$ & $2.54 \pm 0.43$ & $1.56 \pm 0.54$ & 0.87 & 0.831 \\
\hline $\mathrm{NH}_{4}^{+}\left(\mathrm{mg} \mathrm{l}^{-1}\right)$ & $82.77 \pm 19.78$ & $64.85 \pm 19.26$ & $101.80 \pm 36.54$ & $58.14 \pm 8.6$ & 0.71 & 0.869 \\
\hline $\mathrm{PO}_{4}^{-3}\left(\mathrm{mg} \mathrm{l}^{-1}\right)$ & $5.43 \pm 2.84$ & $2.72 \pm 1.11$ & $3.86 \pm 1.28$ & $2.48 \pm 0.06$ & 13.067 & 0.004 \\
\hline
\end{tabular}

Los valores son la media \pm error estándar. Concentración de amonio $\left(\mathrm{NH}_{4}{ }^{+}\right)$; concentración de fosfatos $\left(\mathrm{PO}_{4}^{-3}\right)^{-3}$; concentración de nitratos más nitritos $\left(\mathrm{NO}_{2}{ }^{-}+\mathrm{NO}_{3}{ }^{-}\right)$; concentración de sulfuro $\left(\mathrm{S}^{-2}\right)$. Los valores de Kruskal-Wallis corresponde el análisis entre sitios.

TABLA 4. Hidroperíodo de los sitios de manglar de Bahamitas en Laguna de Términos en el período de estudio.

\begin{tabular}{|c|c|c|c|c|c|c|}
\hline Variables & Sitio 1 & Sitio 2 & Sitio 3 & Sitio 4 & $\begin{array}{c}\text { Kruskal-Wallis } \\
\text { test }\end{array}$ & Valor de $P$ \\
\hline $\begin{array}{l}\text { Frecuencia } \\
\left(\# \text { mareas } \text { mes }^{-1} \text { ) }\right.\end{array}$ & $2.58 \pm 0.75$ & $3.66 \pm 1.36$ & $13.25 \pm 1.66$ & $12.41 \pm 1.53$ & 28.81 & $<0.001$ \\
\hline Periodo (h mes ${ }^{-1}$ ) & $695.11 \pm 14.81$ & $631.36 \pm 44.88$ & $339 \pm 78.27$ & $334.20 \pm 66.00$ & 22.63 & $<0.001$ \\
\hline Nivel de inundación (m) & $0.16 \pm 0.01$ & $0.25 \pm 0.03$ & $0.084 \pm 0.01$ & $0.086 \pm 0.01$ & 20.53 & $<0.001$ \\
\hline
\end{tabular}

\section{Proceso de la regeneración natural y su relación} con las variables ambientales

La densidad de plántulas mostró una relación negativa con la concentración de $\mathrm{NH}_{4}{ }^{+}$en el suelo $\left(\mathrm{r}^{2}=0.86, \mathrm{p}<0.05\right.$, Fig. 3A). También se observó una tendencia negativa de la densidad de plántulas en función de la concentración de $\mathrm{S}^{-2}$ (Fig. 3B). Por otro lado, la sobrevivencia de las plántulas fue mayor en sitios con concentraciones más altas de $\mathrm{PO}_{4}^{-3}\left(\mathrm{r}^{2}=0.98, \mathrm{p}<0.05\right.$, Fig. 2C). En forma similar, la temperatura del agua intersticial y la sobrevivencia de las plántulas mostraron una relación positiva $\left(\mathrm{r}^{2}=0.98, \mathrm{p}<0.05\right.$, Fig. $\left.3 \mathrm{D}\right)$.

$\mathrm{El}$ análisis NMDS mostró que pueden distinguirse tres grupos con similitudes cercanas a $93 \%$ en sus características biológicas (densidad, altura, TRCA y sobrevivencia), que pueden indicar las posibles trayectorias de los sitios restaurados de acuerdo con el reclutamiento observado (Fig. 4). Los sitios 1 y 3 conformaron un grupo, pero compartieron similitudes con el sitio 4. El sitio 2, por sí mismo, conformó otra agrupación; este sitio de manglar es de tipo cuenca y la regeneración presentó valores bajos de densidad, altura y tasa de crecimiento en comparación con los otros sitios en restauración (Tabla 2). Por último, un tercer grupo fue conformado por el sitio 4 donde las características de la estructura de la vegetación fueron de manglares de cuenca (Tabla 1). 

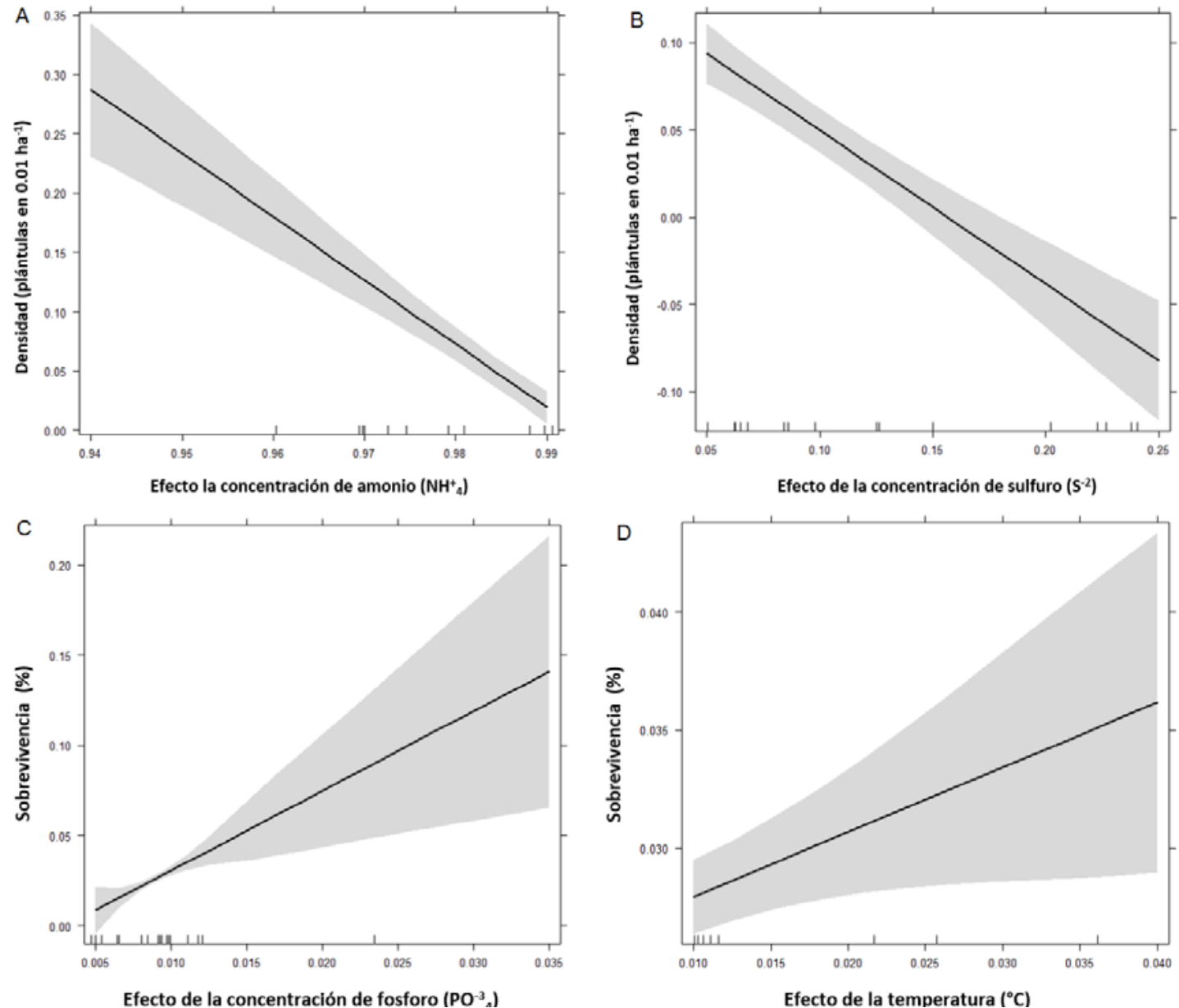

FIGURA 3. Efecto de la concentración de $\mathrm{NH}_{4}^{+}(\mathrm{A})$ y S $\mathrm{S}^{-2}(\mathrm{~B})$ sobre la densidad de plántulas y de la concentración de $\mathrm{PO}_{4}^{-3}(\mathrm{C})$ y de la temperatura (D) del agua intersticial sobre la sobrevivencia de plántulas.

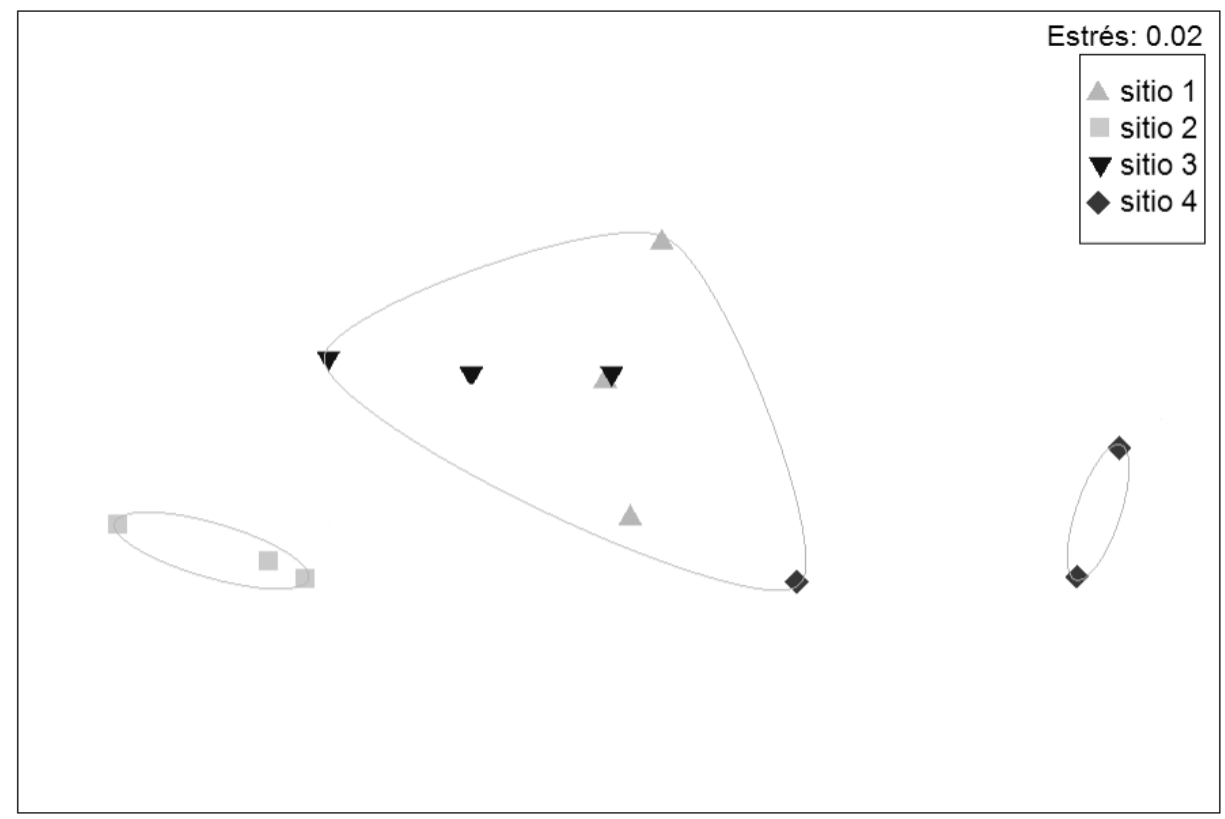

FIGURA 4. Diagrama del escalamiento multidimensional no métrico (NMDS) de las características de la regeneración natural, en sitios con diferentes etapas de restauración ecológica. 


\section{DISCUSIÓN}

La restauración hidrológica en el estero Bahamitas, modificó el patrón del hidroperíodo en las áreas degradadas, relacionándose con el tiempo de la restauración. El resultado de la reconexión hidrológica fue el incremento de la dispersión y la incorporación de especies de manglar a las áreas de restauración. En un principio, cuando los niveles de inundación fueron menores (mes de mayo), el establecimiento de las especies fue dominado por A. germinans; R. mangle se estableció durante el período de mayor inundación (mes de octubre) y $L$. racemosa lo hizo con inundaciones intermedias (mes de febrero). Se ha determinado que la diversidad y la estructura forestal de los sitios naturales de manglar son un factor clave en el proceso de recuperación de la regeneración natural en áreas degradadas y adyacentes a estos sitios. La estructura forestal de árboles en el sitio 1 fue representada por las tres especies de manglar; entre ellas $R$. mangle fue la que registró mayor densidad de árboles (Tabla 1); a pesar de esto, el número de plántulas de esta especie en el área de restauración fue menor en comparación con $A$. germinans. Las diferencias en la dispersión de las especies de manglar determinaron la regeneración en las zonas degradadas. Otros estudios determinaron que la marea tiene un efecto diferencial en la dispersión de propágulos y establecen que los propágulos más pequeños pueden dispersarse a mayor distancia de los árboles madre (Sousa, Kennedy, Mitchell y Ordóñez, 2007). En el área de restauración, A. germinans fue la que mejor se dispersó debido al tamaño de sus propágulos que les permitió moverse con las inundaciones en toda el área (McKee, 1995; Sousa et al., 2007, 2003). Con este estudio se estableció que es necesario mantener y rehabilitar los canales de marea para restablecer la comunicación entre las zonas de manglar natural y el manglar degradado para facilitar el establecimiento de las diferentes especies de manglar.

La restauración hidrológica cambió positivamente las condiciones fisicoquímicas del suelo, debido al ingreso y salida frecuente de agua de la laguna hacia las zonas de restauración a través de los canales de marea rehabilitados; las variables ambientales de los sitios de restauración presentaron valores cercanos al sitio de referencia (Tabla 3). En el caso de la salinidad del agua intersticial, los sitios en restauración fueron ligeramente menores a 49 ups, en comparación con los registrados en el sitio de referencia (55 ups). Adicionalmente, la concentración máxima de $\mathrm{S}^{-2}$ fue de $24.78 \mathrm{mg} / 1$, menor a los niveles determinados en otras islas cársticas con valores entre $32 \mathrm{mg} / \mathrm{l}$ y $128 \mathrm{mg} / \mathrm{l}$ Mckee (1993); los cuales representan niveles tóxicos para $A$. germinans y R. mangle. El aumento de la frecuencia de inundación, como resultado de la restauración, favoreció la oxidación de los sulfuros por la incorporación del oxígeno al suelo, disminuyendo los niveles de concentración del S-2. El mejoramiento de las condiciones fisicoquímicas en relación con el tiempo de restauración ayudó al establecimiento de $L$. racemosa hacia el final del estudio, es por esto que la densidad de esta especie fue menor en comparación con las otras. Este proceso de colonización ha sido observado en otros humedales en condiciones de degradación (McKee, 1995).

El modelo lineal mixto demostró que la variación espacial de la colonización del manglar estuvo en función de la fuerza hidrológica que transportó propágulos desde el sitio de referencia hacia el interior de los sitios en restauración. Las tasas más altas de reclutamiento para $A$. germinans y R. mangle se observaron dentro de los primeros $100 \mathrm{~m}$ de distancia respecto al manglar natural. El desarrollo de la estructura forestal de los árboles madre determinó la vigorosidad de los propágulos que fueron clave para la dispersión (Amir, 2012; Clarke, 2004); para el establecimiento de estos propágulos son importantes las condiciones del agua y el suelo de cada sitio (Ravishankar \& Ramasubramanian, 2004). Los resultados de este estudio sustentaron la necesidad de incluir sitios naturales y en buen estado de su estructura forestal en el desarrollo de proyectos de restauración.

Las áreas en restauración (desprovistas de árboles) registraron las mayores temperaturas del agua intersticial, pero no hubo diferencias significativas entre los sitios; sin embargo, hubo relación positiva de la sobrevivencia de las 
plántulas con la temperatura. Esta relación se explica por la interacción de esta variable con otros agentes modulares como la irradiación solar, luz y movimiento del agua. Las mayores sobrevivencias fueron registradas en los sitios en restauración, en contraste con el sitio de referencia, donde se presentó la mayor densidad de plántulas de $A$. germinans y R. mangle. A pesar de ello, solo se registraron plántulas con alturas menores a $40 \mathrm{~cm}$ y valores de sobrevivencia por debajo de $70 \%$. La falta de crecimiento en altura de las plántulas se explica por la disponibilidad de recursos como luz y nutrientes (Berger y Hildenbrandt, 2000); y por la competencia intra e interespecífica de las plantas (Maestre, Callaway, Valladares y Lortie, 2009).

En la relación de los nutrientes con el desarrollo de las plántulas se observó una tendencia positiva. $\mathrm{El} \mathrm{NH}_{4}{ }^{+}$es el principal nutriente que utilizan las plántulas, aproximadamente $90 \%$ de amonio es absorbido a través del sistema de raíces (Alongi, 1996). Este nutriente influyó directamente en el aumento de la población de plántulas de manglar en los sitios restaurados. $\mathrm{El} \mathrm{PO}_{4}^{-3}$ es reconocido como un elemento limitante del desarrollo estructural de los manglares (Lovelock, Feller, Ball, Engelbrecht y Ewe, 2006; Naidoo, 2006). En este estudio, las plantas con mayor altura y sobrevivencia fueron registradas en los sitios donde las concentraciones de $\mathrm{PO}_{4}^{-3}$ fueron mayores. Los sitios con mayor tiempo de restauración registraron niveles de $\mathrm{PO}_{4}^{-3}$ más cercanos a los del sitio de referencia. La reconexión hidrología de las áreas de restauración con la Laguna de Términos favoreció la importación de subsidios de nutrientes a través de las mareas (Rivera-Monroy, Day, Twilley, Vera-Herrera y Coronado-Molina, 1995).

El análisis de NMDS permitió identificar tres agrupaciones de los sitios con base en el comportamiento de la regeneración natural: intermedio, referencia y divergente. En este trabajo se identificó que los sitios 1 y 3 presentaron características de regeneración intermedia, con densidades entre 111 y 82 plántulas en 0.01 ha y alturas entre $44 \mathrm{~cm}$ y $55 \mathrm{~cm}$, por lo cual presentan una trayectoria de recuperación hacia un hábitat muy similar al sitio de referencia (Suding y Hobbs, 2009). En contraste, el sitio 2 tomó una trayectoria divergente (Suding, 2011), la cual se asocia con una pobre estructura forestal, bajas densidades y menor altura de plántulas. La divergencia en este sitio se debe a que tiene diferentes condiciones fisicoquímicas. El sitio 2 presentó mayores valores de salinidad y permaneció más tiempo inundado, lo cual influyó sobre la dispersión y el reclutamiento. Es posible que este sitio esté desviándose hacia otro estado, lo que podría resultar en un hábitat regenerado pero distinto al sitio de referencia y seguir una trayectoria del paisaje diferente a lo esperado (Matthews, Spyreas y Enderess, 2009; Weinstein, Teal, Balletto y Strait, 2001). La altura se vio afectada por la defoliación causada por insectos; por lo que la herbivoría fue otro factor involucrado en el proceso de reclutamiento, (Monie, Florentine y Palmer, 2013). La divergencia observada podría contribuir a la sostenibilidad del área de estudio debido a la heterogeneidad adicional en el ecosistema, que le confiere resiliencia (Zedler, Doherty y Miller, 2012).

\section{CONCLUSIONES}

Las acciones de restauración reestablecieron la conexión hidrológica entre las zonas de manglar degrado y la Laguna de Términos. Las acciones llevadas a cabo mejoraron las variables fisicoquímicas del suelo, lo que propició el establecimiento e incorporación de nuevas especies de manglar en el área de restauración.

La inclusión de áreas naturales de manglar con buena estructura forestal fue una estrategia clave para la dispersión y reclutamiento de propágulos hacia las zonas de restauración. Los árboles madre fueron proveedores de propágulos de todas las especies presentes en el área, contribuyendo a incrementar la diversidad. La dispersión, establecimiento y crecimiento de plántulas de manglar estuvieron en función de la distancia respecto a los manglares naturales.

Los resultados generados en este estudio respecto a los cambios ambientales y la respuesta de la regeneración natural del manglar contribuyen al conocimiento para la implementación de programas de restauración de manglares. 


\section{RECONOCIMIENTOS}

Se agradece a la Universidad Autónoma del Carmen y al proyecto del Gran Ecosistema del Golfo de México por ser las instituciones responsables de implementar el Programa Piloto de Restauración de manglares en la Isla del Carmen. En especial, a Emma Guevara Carrío por el soporte logístico; a Tomás Zaldívar Jiménez, Mario Alejandro Gómez Ponce, Hernán Álvarez Guillén y Andrés Reda Deara por la colecta de datos de campo. También se agradece a Fermín S. Castillo-Sandoval por el apoyo para los análisis químicos. Se agradece a la Sra. Herminia Rejón Salazar y a la Comunidad de Restauradores del mangar de Isla Aguada por el soporte en el trabajo de campo realizado en las acciones de restauración del área de estudio. Finalmente, se reconoce a la Comisión Nacional Forestal (CA-CONAFOR-UNACAR-GoM LME 2014 - 2016) por el financiamiento otorgado para la realización de las obras y acciones empleado en la restauración del área de estudio; así como al Consejo Nacional de Ciencia y Tecnología (Conacyt) por las Cátedras 849 y 171 asignadas al proyecto 345 denominado "Programa interdisciplinario de investigación de largo plazo en limnología tropical”.

\section{REFERENCIAS}

Alongi, D. M. (1996). The dynamics of benthic nutrient pools and fluxes in tropical mangrove forests. Journal of Marine Research, 54(1), 123-148. doi: 10.1357/002224096321347

Amir, A. A. (2012). Canopy gaps and the natural regeneration of Matang mangroves. Forest Ecology and Management, 269, 6067. doi: 10.1016/j.foreco.2011.12.040

American Public Health Association [APHA] (2005). Standard Methods for the Examination of Water \& Wastewater (21a ed.). Washington DC: APHA.

Aronson, J., \& Alexander, S. (2013). Ecosystem restoration is now a global priority: Time to roll up our sleeves. Restoration Ecology, 21(3), 293-296. doi: 10.1111/rec.12011

Bach, L., Calderon, R., Cepeda, M. F., Oczkowski, A., Olsen, S., \& Robadue, D. (2005). Managing freshwater inflows to estuaries level one site profile: Laguna de Términos and its Watershed, Mexico. Narragansett, RI: Coastal Resources Center, University of Rhode Island.

Barbier, E. B. (2016). The protective service of mangrove ecosystems: A review of valuation methods. Marine Pollution
Bulletin, 109(2), 676-681.

http:/ / doi.org/10.1016/j.marpolbul.2016.01.033

Berger, U., \& Hildenbrandt, H. (2000). A new approach to spatially explicit modelling of forest dynamics: Spacing, ageing and neighbourhood competition of mangrove trees. Ecological Modelling, 132(3), 287-302. doi: 10.1016/S03043800(00)00298-2

Bohn, H. (1971). Redox potential. Soil Science, 112, 39-45.

Brown, B., Fadillah, R., Nurdin, Y., Soulsby, I., \& Ahmad, R. (2014). Community based ecological mangrove rehabilitation (CBEMR) in Indonesia. S.a.p.i.En.S, 7(2), 113.

Buelow, C., \& Sheaves, M. (2015). A birds-eye view of biological connectivity in mangrove systems. Estuarine, Coastal and Shelf Science, 152, 33-43. doi: 10.1016/j.ecss.2014.10.014

Chen, R., \& Twilley, R. R. (1999). A simulation model of organic matter and nutrient accumulation in mangrove wetland soils. Biogeochemestry, 44(1), 93-118.

Clarke, K. R., \& Ainsworth, M. (1993). A method of linking multivariate community structure to environmental variables. Marine Ecology Progress Series, 92(3), 205-219. doi: 10.3354/meps092205

Clarke, P. J. (2004). Effects of experimental canopy gaps on mangrove recruitment: lack of habitat partitioning may explain stand dominance. Journal of Ecology, 92(2), 203-213. doi: 10.1111/j.0022-0477.2004.00861.x

Contreras Ruiz Esparza, A., Douillet, P., \& Zavala-Hidalgo, J. (2014). Tidal dynamics of the Terminos Lagoon, Mexico: Observations and 3D numerical modelling. Ocean Dynamics, 64(9), 1349-1371. doi: 10.1007/s10236-014-0752-3

Comisión Nacional para el Conocimiento y Uso de la Biodiversidad [Conabio] (2007). Cuerpos de agua de México, con descripción y nombre. Escala 1:250,000.

Coronado-Molina, C., Alvarez-Guillen, H., Day, J. W., Reyes, E., Perez, B. C., Vera-Herrera, F., \& Twilley, R. (2012). Litterfall dynamics in carbonate and deltaic mangrove ecosystems in the Gulf of Mexico. Wetlands Ecology and Management, 20(2), 123-136. doi: 10.1007/s11273-012-9249-3

Curtis J. T., \& McIntosh R. P. (1951) An upland forest continuum in the pariré-forest border region of Wisconsin. Ecology, 32(3), 476-496.

Secretaría de Desarrollo Social [Sedesol] (6 de junio de 1994). Decreto por el que se Declara como Área Natural Protegida con el Carácter de Protección de Flor y Fauna conocida como Laguna de Términos, ubicada en los municipios de Carmen, Palizada y Champotón, Estado de Campeche. 
Diario Oficial de la Federación, pp. 58-64 Ciudad de México, México.

Duke, N. C. (2001). Gap creation and regenerative processes driving diversity and structure of mangrove ecosystems. Wetlands Ecology and Management, 9, 257-269. doi: 10.1023/A:1011121109886

Gedan, K. B., Kirwan, M. L., Wolanski, E., Barbier, E. B., \& Silliman, B. R. (2011). The present and future role of coastal wetland vegetation in protecting shorelines: answering recent challenges to the paradigm. Climatic Change, 106(1), 729. doi: $10.1007 /$ s10584-010-0003-7

Guerra-Santos, J. J., Kahl, J. D. W. (2017). Redefining the Seasons in the Términos Lagoon Region of Southeastern México: May Is a Transition Month, Not a Dry Month. Journal of Coastal Research, 34(1), 193-201. doi: 10.2112/JCOASTRESD-16-00114.1

Hastuti, E. D., Anggoro, S., \& Pribadi, R. (2012). The effects of environmental factors on the dynamic growth pattern of mangrove Avicennia marina. Journal of Coastal Development, 16(1), 57-61.

Jaramillo, F., Licero, L., Åhlen, I., Manzoni, S., RodríguezRodríguez, J. A., Guittard, A., \& Espinosa, L. F. (2018). Effects of hydroclimatic change and rehabilitation activities on salinity and mangroves in the Ciénaga Grande de Santa Marta, Colombia. Wetlands, 38(4) 755-767. doi: 10.1007/s13157-018-1024-7

Kuenzer, C., Bluemel, A., Gebhardt, S., Quoc, T. V., \& Dech, S. (2011). Remote Sensing of Mangrove Ecosystems: A Review. Remote Sensing, 3(12), 878-928. doi: $10.3390 /$ rs 3050878

Lewis, R. R., Milbrandt, E. C., Brown, B., Krauss, K. W., Rovai, A. S., Beever, J. W., \& Flynn, L. L. (2015). Stress in mangrove forests: Early detection and preemptive rehabilitation are essential for future successful worldwide mangrove forest management. Marine Pollution Bulletin, 109(2), 764-771. doi: 10.1016/j.marpolbul.2016.03.006

Lovelock, C. E., Feller, I. C., Ball, M. C., Engelbrecht, B. M. J., \& Ewe, M. L. (2006). Difference in plant function in phosphorous- and nitrgoen- limited mangrove ecosystems. New Pbytologist, 172(3), 514-522.

Lugo, A. E., \& Snedaker, S. C. (1974). The Ecology of Mangroves. Annual Review of Ecology and Systematics, 5(1), 3964. doi: 10.1146/annurev.es.05.110174.000351

Maestre, F. T., Callaway, R. M., Valladares, F., \& Lortie, C. J. (2009). Refining the stress-gradient hypothesis for competition and facilitation in plant communities. Journal of
Ecology, $\quad 97(2), \quad$ 199-205. doi: 10.1111/j.13652745.2008.01476.x

Matthews, J. W., Spyreas, G., \& Endress, A. G. (2009). Trajectories of vegetation-based indicators used to assess wetland restoration progress. Ecological Applications, 19(8), 2093-2107. doi: 10.1890/08-1371.1

McKee, K. L. (1993). Soil Physicochemical Patterns and Mangrove Species Distribution, Reciprocal Effects? Journal of Ecology, 81(3), 477-487. doi: 10.2307/2261526

McKee, K. L. (1995). Seedling recruitment patterns in a Belizean mangrove forest: effects of establishment ability and physico-chemical factors. Oecologia, 101(4), 448-460. doi: 10.1007/BF00329423

McKee, K. L., Mendelssohn, I. A., \& Hester, M. W. (1988). Reexamiation of the porewater sulfide concentrations and redox potencial near the areal roots of Rhizophora mangle and Avicennia germinans. American Journal of Botany, 75(9), 1352-1359. doi: 10.2307/2444458

Monie, K., Florentine, S., \& Palmer, G. (2013). Recruitment and functionality traits as bioindicators of ecological restoration success in the Lurg Hills district, Victoria, Australia. Ecological Processes, 2(1), 27. doi: 10.1186/2192-1709-2-27

Naidoo, G. (2006). Factors contributing to dwarfing in the mangrove Avicennia marina. Annals of Botany, 97(6), 10951101. doi: $10.1093 / \mathrm{aob} / \mathrm{mcl} 064$

Pérez-Ceballos, R., Echeverria-Avila, S., Zaldívar-Jiménez, A., Zaldívar-Jiménez, T., \& Herrera-Silveira, J. (2017). Contribution of microtopography and hydroperiod to the natural regeneration of Avicennia germinans in a restored mangrove forest. Ciencias Marinas, 43(1), 55-67. doi: $10.7773 / \mathrm{cm} . v 43 i 1.2683$

Quinn, G., \& Keough, J. M. (2002). Experimental design and data analysis for biologists. Cambridge University Press, 277(2), 197-198. doi: 10.1016/S0022-0981(02)00278-2

R Development Core Team, R. (2008). R: A Language and Environment for Statistical Computing. $\mathrm{R}$ Foundation for Statistical Computing. Computing, Vienna, Austria. doi: 10.1007/978-3-540-74686-7

Ravishankar, T., \& R. Ramasubramanian. (2004). Manual on mangrove nursery techniques. Chennai, India: M.S. Swaminathan Research Foundation.

Rivera-Monroy, V. H., Day, J., Twilley, R. R., Vera-Herrera, F., \& Coronado-Molina, C. (1995). Flux of nitrogen and sediment in a fringe mangrove forest in Terminos Lagoon. Estuarine, Coastal and Shelf Science, 40(2), 139-160. doi: 10.1016/S02727714(05)80002-2 
Rodríguez-Ramírez, A., Nivia-Ruíz, J., \& Garzón-Ferreira, J. (2004). Structural and functional characteristics of Avicennia germinans mangrove formation of Chengue bay (Colombian Caribbean). Boletin de Investigaciones Marinas y Costeras, 33(1), 223-244.

Salmo, S. G., Lovelock, C., \& Duke, N. C. (2013). Vegetation and soil characteristics as indicators of restoration trajectories in restored mangroves. Hydrobiologia, 720(1), 1-18.

Sousa, W. P., Kennedy, P. G., Mitchell, B. J., \& Ordóñez L, B. M. (2007). Supply-side ecology in mangroves: Do propagule dispersal and seedling establishment explain forest structure? Ecological Monographs, 77(1), 53-76. doi: $10.1890 / 05-1935$

Sousa, W. P., Quek, S. P., \& Mitchell, B. J. (2003). Regeneration of Rhizophora mangle in a Caribbean mangrove forest: Interacting effects of canopy disturbance and a stem-boring beetle. Oecologia, 137(3), 436-445. doi: 10.1007/s00442-003$1350-0$

Suding, K. N. (2011). Toward an era of restoration in ecology: successes, failures, and opportunities ahead. Annual Review of Ecology, Evolution, and Systematics, 42, 465-487. doi: 10.1146/annurev-ecolsys-102710-145115

Suding, K. N., \& Hobbs, R. J. (2009). Models of ecosystem dynamics as frameworks for restoration ecology. En K. N. Hobbs, \& R. J. Suding (Eds.), New models for ecosystem dynamics and restoration (1a ed., pp. 3-21). Washington DC: Island Press.

Turner, R. E., \& Lewis III, R. R. (1997). Hydrologic restoration of coastal wetlands. Wetlands Ecology and Management, 4(2), 65-72. doi: 10.1007/BF01876229

Tomlinson, P. B. (1986). The Botany of Mangroves. Cambridge Univ. Press.

Twilley, R. R., \& Rivera-Monroy, V. H. (2005). Developing performance measures of mangrove wetlands using simulation models of hydrology, nutrient biogeochemistry, and community dynamics. Journal of Coastal Research, SI 40, 79-93. doi: $10.2307 / 25736617$

Venegas P., Y. (Comp.) (2003). Ficha informativa de los humedales de Ramsar. Área de protección de flora y fauna Laguna de Términos, Campeche, México. Recuperado de http://www.conanp.gob.mx/conanp/dominios/ramsar/d ocs/sitios/FIR_RAMSAR/Campeche/APFF_Laguna_de_ Terminos/Laguna $\% 20 \mathrm{de} \% 20 \mathrm{~T} \% \mathrm{C} 3 \%$ A 9 rminos.pdf
Vogt, J., Lin, Y., Pranchai, A., Frohberg, P., Mehlig, U., \& Berger, U. (2014). The importance of conspecific facilitation during recruitment and regeneration: A case study in degraded mangroves. Basic and Applied Ecology, 15(8), 651-660. doi: 10.1016/j.baae.2014.09.005

Vovides, A. G., Bashan, Y., López-Portillo, J. A., \& Guevara, R. (2011). Nitrogen Fixation in Preserved, Reforested, Naturally Regenerated and Impaired Mangroves as an Indicator of Functional Restoration in Mangroves in an Arid Region of Mexico. Restoration Ecology, 19(201), 236-244. doi: 10.1111/j.1526-100X.2010.00713.x

Weinstein, M. P., Teal, J. M., Balletto, J. H., \& Strait, K. A. (2001). Restoration principles emerging from one of the world's largest tidal marsh restoration projects. Wetlands Ecology and Management, 9(5), 387-407. doi: 10.1023/A:1012058713910

Zaldívar-Jiménez, A., Ladrón de Guevara-Porras, P., PérezCeballos, R., Díaz-Mondragón, S., \& Rosado-Solórzano, R. (2017). US-Mexico joint Gulf of Mexico large marine ecosystem based assessment and management: Experience in community involvement and mangrove wetland restoration in Términos lagoon, Mexico. Environmental Development, 22, 206-213. doi: 10.1016/j.envdev.2017.02.007

Zedler, J. B., Doherty, J. M., \& Miller, N. A. (2012). Shifting restoration policy to address landscape change, novel ecosystems, and monitoring. Ecology and Society, 17(4), 36. doi: 10.5751/ES-05197-170436

Zhao, Q., Bai, J., Huang, L., Gu, B., Lu, Q., \& Gao, Z. (2016). A review of methodologies and success indicators for coastal wetland restoration. Ecological Indicators, 60, 442-452. doi: 10.1016/j.ecolind.2015.07.003

Manuscrito recibido el 3 de abril de 2018

Aceptado el 31 de julio de 2018

Publicado el 29 de julio de 2019

Este documento se debe citar como:

Echeverría-Ávila, S., Pérez-Ceballos, R., Zaldívar-Jiménez, A., CanalesDelgadillo, J., Brito-Pérez, R., Merino-Ibarra, M., \& y Vovides, A. (2019). Regeneración natural de sitios de manglar degradado en respuesta a la restauración hidrológica. Madera y Bosques, 25(1), e2511754. doi: 10.21829/myb.2019.2511754

Madera y Bosques por Instituto de Ecología, A.C. se distribuye bajo una Licencia Creative Commons Atribución-NoComercialCompartirlgual 4.0 Internacional. 